\title{
Computing adrenal uptakes with compact, fixed-size regions
}

\author{
Kenneth F. Koral *, Hany Abukhadra, Michael Tuscan and William H. Beierwaltes \\ Department of Internal Medicine, Division of Nuclear Medicine Box No. 21, R-3054 Kresge II, University of Michigan Medical Center, \\ Ann Arbor, MI 48109, USA
}

\begin{abstract}
In planar nuclear medicine imaging of the adrenal gland, there are three requirements for an uptake determination: finding the region of interest, subtracting 'background', and converting cps to $\mu \mathrm{Ci}$ : A computer program that covers each requirement is outlined. The region of interest is determined by a nearest-neighbor region-growing technique. It starts from an operator-chosen center, searches for the hottest pixels, and terminates when a fixed-size region is found. If the resulting region is unacceptable, recourse to a second level of searching, which produces a more compact region, is possible. Background from underlying and overlying tissue is estimated from the average counts/pixel in a narrow 'ring' surrounding the adrenal. Conversion to $\mu \mathrm{Ci}$ is carried out by use of a calibration equation which requires a value for the adrenal depth. This value may be obtained from a lateral view.

The program is available in listing form. For use with a Medical Data Systems $A^{2}$ computer, files can be transferred directly. Parameters which should be changed for the use of the program with another camera-collimator system are noted.
\end{abstract}

Background subtraction Nuclear medicine Region-growing Region of interest Uptake

\section{INTRODUCTION}

In planar nuclear medicine imaging of the adrenal glands, whether a cortical $[1,2]$ or a medullary [3] gamma-emitting radioisotope is used, quantification of the uptake requires:

(i) Determining the region of interest in the fixed-time projection image.

(ii) Summing the counts within that region of interest and subtracting an estimate of the 'background' which is included.

(iii) Relating the resultant number of counts detected in a fixed time to the number of $\mu \mathrm{Ci}$ of radioisotope actually taken up by the gland or tumor. Such a calibration involves taking into account the effects of absorption and scattering of the gamma-rays as they emerge from the body.

Adrenal images generally are low contrast because of uptake in the liver and in body tissue and

* To whom reprint requests should be addressed are noisy because usually $<1 \%$ of the dose comes to reside in the glands or tumor. These image features make it very difficult to use edge detection algorithms to delineate the region of interest.

In this paper, we will outline an approach to the uptake problem that has been in use at the Univei sity of Michigan since it was introduced 5 years ago [4]. This approach now uses a region-growing algorithm that has two levels. At level 1, the algorithm searches out the 'hottest' pixels near a starting pixel to be categorized as adrenal points. It sometimes happens that the adrenal is poorly resolved from an adjacent structure and this level 1 searching includes obviously non-adrenal areas. Two years ago a second level of searching was added to cover this case. At level 2, a further constraini toward circular regions is imposed. This constraint is only invoked if level 1 searching fails because if it was used for all adrenals, the tendency would be to avoid prolate shapes in favor of circular shapes even in cases where the adrenal is well resolved from nearby structures. Recently the 
entire program has been ajapied to a Medical Data Systems (MDS) $\mathrm{A}^{2}$ computer and the routines are now available.

\section{METHODS}

The basic image for determining adrenal uptake is a 20 min posterior image taken with an Anger scintillation camera and a high-energy parallel-hole collimator. The region of interest is determined by a nearest-neighbor region-growing technique. The starting point for the region growth is a center chosen by thic operator; then the computer takes over. eventually stopping the growth after a fixed number of matrix points has been selected as belonging to the adrenal. The number of counts within the region is found by summation and the 'background' within the region from underlying and overlying tissue is estimated by finding a value for background counts/pixel. This value is determined from points surrounding the adrenal image, under the assumption that the background across the image is smoothly varying. This assumption, that there is no depression in the background profile at the location of the glands due to a deficit of background-emitting tissue, is reasonable because the adrenals are small compared to the body thickness. Such a depression has been reported for heart planar imaging [5]; however. that organ is a much larger fraction of the body thickness than is the adrenal. The calibration method that allows conversion of cpm to uptake in $\mu \mathrm{Ci}$ is based upon a phantom measurement $[1,6]$. It is necessary to assume an adrenal cepth or to take a lateral image with a radioactive marker placed on the back to determine the depth.

\subsection{Cumpact, fixed-size region of interest}

The starting point for the active part of the algorithm involves the operator sequentially positioning an $x$-axis and $y$-axis cursor over the center of the pertinent adrenal (uptakes for a single adrenal or a pair of adrenais can be found). The program then categorizes this pixel as part of the main adrenal (category 1) and the 8 nearestneighbor pixels as tentatively being adrenal edge elements (category 2 ). It then checks that the current number of adrenal pixels ( 9 at this point) is not greater than or equal to the predetermined size number. If it is not, it checks all category 2 elements for the one or more with the largest number of counts. These elements are updated to category 1 and their 8 nearest neighbors are added to the adrenal (in category 2) if they are not already categorized. The algorithm repeats in this way until the predetermined number of pixels is achieved. (A slight variation is required at the endpoint so that the number is attained exactly.) Figure 1 shows the progress of the region growing over 3 cycles for a given data set. For the example, 20 pixels are to be found and the algorithm reaches this number after exactly 3 cycles. (Thus, the endpoint algorithm variation is not illustrated.)

At this point in the program, the image is displayed with the selected adrenal region designated by a surrounding black band and the operator is asked if the region is acceptable. Usually the region is quite compact but it can feature a 'flare' going off in a particular direction. This occurs, for instance, when the right adrenal merges with the liver and the algorithm tracks into the liver region and stays there. (This is common in dexamethasone-suppression adrenal scanning; see the example in [2].) If the search teatures such a flare and thus has failed, the operator can return that the result is unacceptable and the algorithm goes to a second level of searching. This level is like the first except that the algorithm chooses new adrenal points not on the basis of counts alone but on the basis of counts inversely weighted by the square root of the distance from the adrenal center. At the end of this searching, the chosen region is again displayed and the operator queried as to its acceptability. (As a modification of the program, the exact power of the distanse can be varied to more or less guarantee a selected region that is compactly located around the center which was originally chosen by the operator.)

The fixed number of pixels which terminates either level of searching corresponds to an area of $17.3 \mathrm{~cm}^{2}$ (108 4-mm-square pixels). The choice was made empirically as that number which, at level 1 , led to regions that included as much of the adrenal as possible without allowing the region to track 


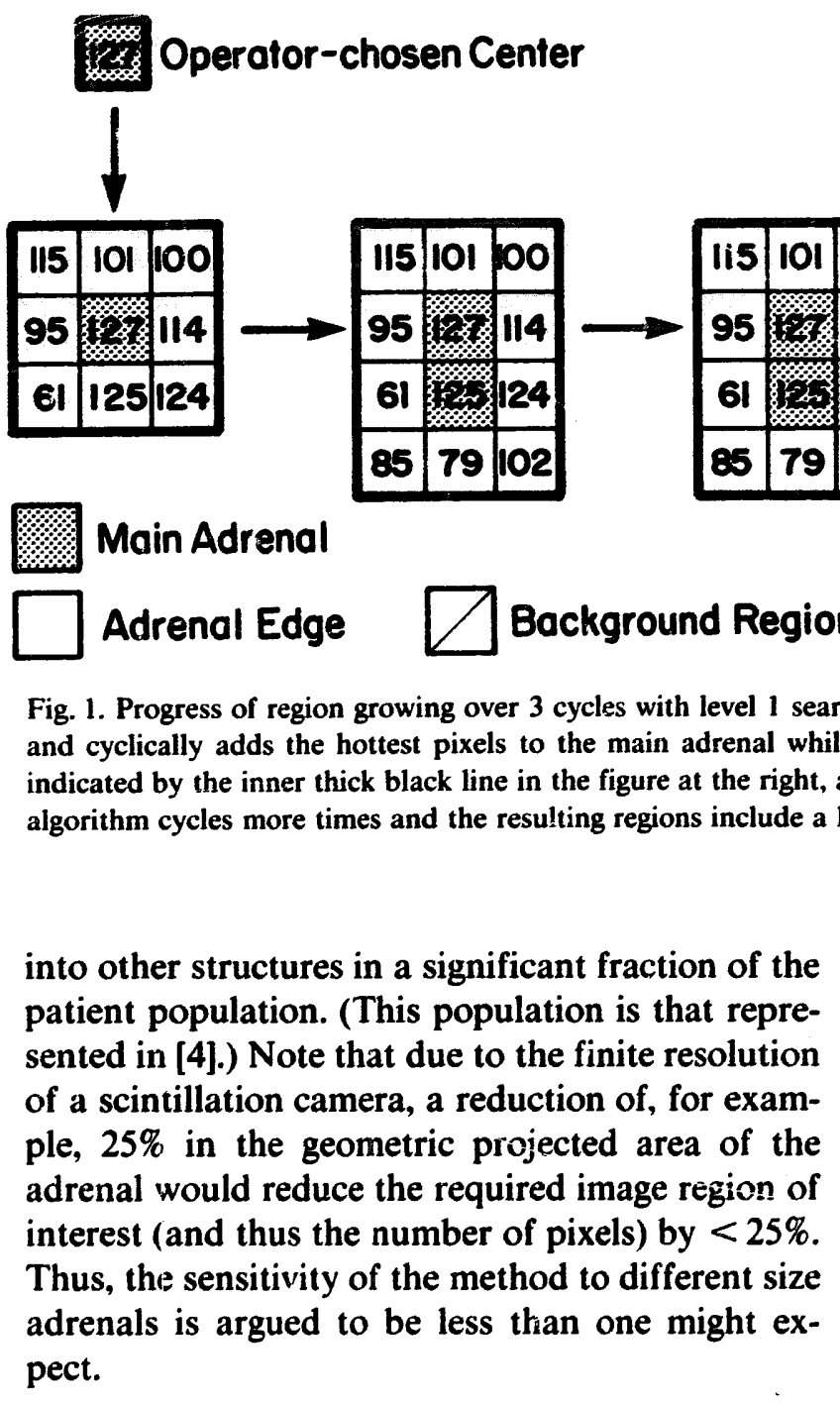

\subsection{Background estimate}

After the region of interest is selected, the algorithm finds the region to be used for estimating background by expansion from the adrenal edge points (category 2). The 24 first and second nearest neighbors of each of these points are assigned to the background estimate region (category 3 ) if they are not already categorized (see right-handside of fig. 1). (It is the background region which forms the irregular dark ring around the region of interest to delineate it for display.) The counts in the background estimate region are then averaged to obtain a background counts/pixel which is effectively subtracted from each counts/pixel value in the region of interest. The uptake in counts is then the sum of the corrected values in the region.

\subsection{Calibration}

Before the uptake in counts is converted to one in $\mu \mathrm{Ci}$. an assumed value for the depth of the adrenals in $\mathrm{cm}$ is accepted or recourse is made to a lateral image on which cursors can be placed by eye over the adrenal pair and over a marker on the skin so that depth can be calculated. The calculated depth equals the distance in pixel times the camera scale in $\mathrm{mm} /$ pixel. We currently use only a single depth for both adrenals even though measurements on normal subjects have indicated that the left adrenal is an average of $1.8 \mathrm{~cm}$ deeper than the right [7,8]. A change in the program could easily take such a difference into account if there were an easily applied technique for determining it for each patient. This depth calculation, as well as the region of interest conversion from the fixed area in $\mathrm{cm}^{2}$ to number of pixels, requires a previous measurement of the camera scale. Our current value for this scale is $5.0 \mathrm{~mm} /$ pixel for $64 \times 64$ acquire. Changing this value requires a program modification and recompilation.

Given a determination of the adrenal depth, the 


\section{ADRESAL UPTANE}

THE GNIVERSITY OF MCHIGAN HOSPITAL

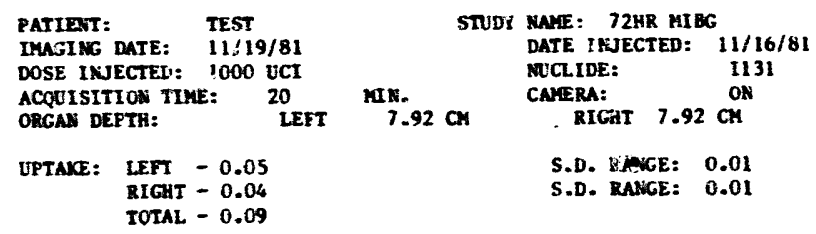

Fig. 2. Sample output: Uptake and ISD are given in units of $\%$ of administered dose corrected for decay to date of imaging.

conversion to uptake in $\mu \mathrm{Ci}$ is carried out as outlined below: For a 1000 hole medium-energy collimator and a $20 \%$ window, a phantom measurement [1,6] established that the effects of attenuation and scatter are accounted for by an exponential equation:

$R=\frac{1}{\alpha} S \mathrm{e}^{-\mu}$

where $R$ is the count rate in cpm, $S$ is the source strength in $\mu \mathrm{Ci}, \mu$ is an effective absorption coeffi-

TABLE I

\begin{tabular}{|c|c|c|}
\hline $\begin{array}{l}\text { File } \\
\text { name }\end{array}$ & $\begin{array}{l}\text { Subroutine } \\
\text { name }\end{array}$ & Function \\
\hline UPTAKE * & MAIN & $64 \times 64$ image mode \\
\hline UP128 & MAIN & $128 \times 128$ byte inage mode \\
\hline BACKPLOT & PLOT & Displays image \\
\hline BACKASC & NUMASC & Converts number to ASC II \\
\hline UPIST & NPIST & Provides image specifier table \\
\hline UPFIND & FIND & Unpacks a word \\
\hline LPCQNV & CONVERT & $\begin{array}{l}\text { Converts image from byte } \\
\text { to word }\end{array}$ \\
\hline BACKERR & ERROR & Error handling routine \\
\hline ADUPAN64 & WORK & $\begin{array}{l}\text { Adrenal edge detection } \\
\text { and uptake }\end{array}$ \\
\hline ADUPAN 128 & WORK 128 & Same for 128 version \\
\hline ADUPBN64 & SQUARE & $\begin{array}{l}\text { Checks } 8 \text { nearest neighbor } \\
\text { for adrenal edge assignment }\end{array}$ \\
\hline ADUPBN 128 & SQR12S & Same for 128 version \\
\hline$A D \cup P C N$ & BKGND & $\begin{array}{l}\text { Checks } 24 \text { first and second } \\
\text { uearest neighbors for back- } \\
\text { ground assigment }\end{array}$ \\
\hline ADUPCN128 & BKG128 & Same for 128 version \\
\hline
\end{tabular}

- All file names have suffix .FR cient, $x$ is the depth below the skin surface and $\alpha$ is a suitable proportionality constant. For ${ }^{131} I$ and our camera system, the current $\alpha=0.00429 \mu \mathrm{Ci}$ $\min$ and $\mu$ is $0.10 \mathrm{~cm}^{-1}$, a value between that of water and fat. For a different camera-collimator system, the calibration can be redone. However, we expect the value of $\mu$ is unlikely to change much and the value of $\alpha$ can itself be re-evaluated more simply as follows: One places a syringe containing a known number of $\mu \mathrm{Ci}, S_{0}$, on the collimator face and records the count rate, $R_{0}$. One then solves the equation above for $\alpha$ with $\boldsymbol{x}=\mathbf{0}$ :

$\alpha=\frac{S_{0}}{R_{0}}$

Insertion of a new $\alpha$ again requires program modification and recompilation.

To obtain the $\%$ of dose, the calculated value for $S$ is divided by the initial dose in $\mu \mathrm{Ci}$ (a program input) corrected for decay to the day of the measurement (also a program input).

A sample uutput is shown in fig. 2. The standard deviation range shown at the right contains only the Poisson statistic errors propagated through the calculation. Other errors have not been estimated but would tend to make the error value larger than the one listed. Also, we have noted that varying the size $10 \%$ or $20 \%$ about the empirical value chosen does not have a large effect on the uptake.

\section{HARDWARE AND SOFTWARE SPECIFI- CATIONS}

This program is user interactive and prompts the operator for pertinent data. Two versions are available for the MDS $A^{2}$ user: one for $64 \times 64$ byte or word mode acquisition and one for $128 \times$ 128 byte mode acquistion. A list of the files involved is given in table 1. Patient images are selected from those available on the patient list and the numbers for the posterior and lateral images of interest should be found before entering the program. The $64 \times 64$ version takes $6 \mathrm{~s}$ searching time/adrenal at level 1 , and $10 \mathrm{~s}$ in addition/ 
adrenal for level 2; the $128 \times 128$ version searches for 4-times as many pisints and takes considerably longer than 4-times as long because of repeated unpacking of a word into 2 bytes. The program uses an overlay of the main subroutine and the display subroutine and so fits into the 26 kilowords allocated to one terminal (32000-6000 words for operating system). The main subroutines, $n$ FORTRAN, could be converted to a system other than the MDS $\mathrm{A}^{2}$.

\section{PROGRAM AVAILABILITY}

The programs are available in listing form. For potential MDS users who can supply a 2.5 megabyte front-loading cartridge disk, or 10 in. magnetic tape, the files could be transferred directly.

\section{ACKNOWLEDGEMENTS}

This work was supported by grants (CA-0901505 and CA-32866-02) from the National Cancer Institute, (1-R01-A7 21 477-02 RAD) from the National Institute of Arthritis, Metabolism and Digestive Disease, contract no. E7-76-5-02-2031 from the Department of Energy, and the Nuclear Medicine Research Fund of the University of Michigan.

\section{REFERENCES}

[1] J.H. Thrall, J.E. Freitas and W.H. Beierwaltes, Adrenal scintigraphy, Sem. Nucl. Med. 8 (1978) 23-41.

[2] M.D. Gross, J.E. Freitas, D.P. Swanson, T.J. Brady and W.H. Beierwaltes, The normal dexamethasone-suppression adrenal scintiscan, J. Nucl. Med 20 (1979) 1131-1:35.

[3] T.W. Valk, M.S. Frager, M.D. Gross, J.C. Sisson, D.M. Wieland, D.P. Swanson, T.J. Mangner and W.H. Beierwaltes, Spectrum of pheochromocytoma in multiple endocrine neoplasia: A scintigraphic portrayal using [ ${ }^{131}$ I]iodobenzylguanidine, Ann. Intern. Med. 94 (1981) 762-767.

[4] K.F. Koral and S.D. Sarkar, An operator-independent method for background subtraction in adrenal-uptake measurements: Concise communication, J. Nucl. Med. 18 (1977) 925-928.

[5] K.A. Narahara, G.W. Hamilton, D.L. Williams, and K.L. Gould, Myocardial imaging with thallium-201: An experimental model for analysis of the true myocardial and background image components, J. Nucl. Med. 18 (1977) 781-786.

[6] R. Morita, L.M. Lieberman, W.H. Beierwaltes et al., Percent uptake of ${ }^{131}$ I radioactivity in the adrenal from radioindinated cholesterol, J. Clin. Endocrinol. Metab. 34 (1972) 36-43.

[7] J.E. Freitus, J.H. Thrall, D.P. Swanson, A. Rifai and W.H. Beierwaltes, Normal adrenal asymmetry: Explanation and interpretation, J. Nuc'. Med. 19 (1978) 149-153.

[8] J.P. Montagne, H.Y. Kressel, M. Korobkin and A.A. Moss, Computed tomography of the normal adrenal glands, Am. J. Roentgenol. 130 (1978) 963-966. 